Article

\title{
On Mann Viscosity Subgradient Extragradient Algorithms for Fixed Point Problems of Finitely Many Strict Pseudocontractions and Variational Inequalities
}

\author{
Lu-Chuan Ceng ${ }^{1}$, Adrian Petruşel ${ }^{2,3}$ (i) and Jen-Chih Yao ${ }^{4, *}$ \\ 1 Department of Mathematics, Shanghai Normal University, Shanghai 200234, China; zenglc@shnu.edu.cn \\ 2 Department of Mathematics, Babeş-Bolyai University, Cluj-Napoca 400084; petrusel@math.ubbcluj.ro \\ 3 Academy of Romanian Scientists, Bucharest 050044, Romania \\ 4 Research Center for Interneural Computing, China Medical University Hospital, Taichung 40447, Taiwan \\ * Correspondence: yaojc@mail.cmu.edu.tw
}

Received: 21 August 2019; Accepted: 26 September 2019; Published: 4 October 2019

\begin{abstract}
In a real Hilbert space, we denote CFPP and VIP as common fixed point problem of finitely many strict pseudocontractions and a variational inequality problem for Lipschitzian, pseudomonotone operator, respectively. This paper is devoted to explore how to find a common solution of the CFPP and VIP. To this end, we propose Mann viscosity algorithms with line-search process by virtue of subgradient extragradient techniques. The designed algorithms fully assimilate Mann approximation approach, viscosity iteration algorithm and inertial subgradient extragradient technique with line-search process. Under suitable assumptions, it is proven that the sequences generated by the designed algorithms converge strongly to a common solution of the CFPP and VIP, which is the unique solution to a hierarchical variational inequality (HVI).
\end{abstract}

Keywords: method with line-search process; pseudomonotone variational inequality; strictly pseudocontractive mappings; common fixed point; sequentially weak continuity

MSC: 47H05; 47H09; 47H10; 90C52

\section{Introduction and Preliminaries}

Throughout this article, we suppose that the real vector space $H$ is a Hilbert one and the nonempty subset $C$ of $H$ is a convex and closed one. An operator $S: C \rightarrow H$ is called:

(i) $L$-Lipschitzian if there exists $L>0$ such that $\|S u-S v\| \leq L\|u-v\| \forall u, v \in C$;

(ii) sequentially weakly continuous if for any $\left\{w_{n}\right\} \subset C$, the following implication holds: $w_{n} \rightarrow$ $w \Rightarrow S w_{n} \rightarrow S w ;$

(iii) pseudomonotone if $\langle S u, u-v\rangle \leq 0 \Rightarrow\langle S v, u-v\rangle \leq 0 \forall u, v \in C$;

(iv) monotone if $\langle S u-S v, v-u\rangle \leq 0 \forall u, v \in C$;

(v) $\gamma$-strongly monotone if $\exists \gamma>0$ s.t. $\langle S u-S w, u-w\rangle \geq \gamma\|u-w\|^{2} \forall u, w \in C$.

It is not difficult to observe that monotonicity ensures the pseudomonotonicity. A self-mapping $S: C \rightarrow C$ is called a $\eta$-strict pseudocontraction if the relation holds: $\langle S u-S v, u-v\rangle \leq\|u-v\|^{2}-$ $\frac{1-\eta}{2}\|(I-S) u-(I-S) v\|^{2} \forall u, v \in C$ for some $\eta \in[0,1)$. By [1] we know that, in the case where $S$ is $\eta$-strictly pseudocontractive, $S$ is Lipschitzian, i.e., $\|S u-S v\| \leq \frac{1+\eta}{1-\eta}\|u-v\| \forall u, v \in C$. It is clear that the class of strict pseudocontractions includes the class of nonexpansive operators, i.e., $\|S u-S v\| \leq\|u-v\| \forall u, v \in C$. Both classes of nonlinear operators received much attention and many numerical algorithms were designed for calculating their fixed points in Hilbert or Banach spaces; see e.g., [2-11]. 
Let $A$ be a self-mapping on $H$. The classical variational inequality problem (VIP) is to find $z \in C$ such that $\langle A z, y-z\rangle \geq 0 \forall y \in C$. The solution set of such a VIP is indicated by $\operatorname{VI}(C, A)$. To the best of our knowledge, one of the most effective methods for solving the VIP is the gradient-projection method. Recently, many authors numerically investigated the VIP in finite dimensional spaces, Hilbert spaces or Banach spaces; see e.g., [12-20].

In 2014, Kraikaew and Saejung [21] suggested a Halpern-type gradient-like algorithm to deal with the VIP

$$
\left\{\begin{array}{l}
v_{k}=P_{C}\left(u_{k}-\ell A u_{k}\right), \\
C_{k}=\left\{v \in H:\left\langle u_{k}-\ell A u_{k}-v_{k}, v_{k}-v\right\rangle \geq 0\right\} \\
w_{k}=P_{C_{k}}\left(u_{n}-\ell A v_{k}\right), \\
u_{k+1}=\varrho_{k} u_{0}+\left(1-\varrho_{k}\right) w_{k} \quad \forall k \geq 0
\end{array}\right.
$$

where $\ell \in\left(0, \frac{1}{L}\right),\left\{\varrho_{k}\right\} \subset(0,1), \lim _{k \rightarrow \infty} \varrho_{k}=0, \sum_{k=1}^{\infty} \varrho_{k}=+\infty$, and established strong convergence theorems for approximation solutions in Hilbert spaces. Later, Thong and Hieu [22] designed an inertial algorithm, i.e., for arbitrarily given $u_{0}, u_{1} \in H$, the sequence $\left\{u_{k}\right\}$ is constructed by

$$
\left\{\begin{array}{l}
z_{k}=u_{k}+\varrho_{k}\left(u_{k}-u_{k-1}\right) \\
v_{k}=P_{C}\left(z_{k}-\ell A z_{k}\right) \\
C_{k}=\left\{v \in H:\left\langle z_{k}-\ell A z_{k}-v_{k}, v_{k}-v\right\rangle \geq 0\right\} \\
u_{k+1}=P_{C_{k}}\left(z_{n}-\ell A v_{k}\right) \quad \forall k \geq 1
\end{array}\right.
$$

with $\ell \in\left(0, \frac{1}{L}\right)$. Under mild assumptions, they proved that $\left\{u_{k}\right\}$ converge weakly to a point of $\operatorname{VI}(C, A)$. Very recently, Thong and Hieu [23] suggested two inertial algorithms with linear-search process, to solve the VIP for Lipschitzian, monotone operator $A$ and the FPP for a quasi-nonexpansive operator $S$ satisfying a demiclosedness property in $H$. Under appropriate assumptions, they proved that the sequences constructed by the suggested algorithms converge weakly to a point of $\operatorname{Fix}(S) \cap \operatorname{VI}(C, A)$. Further research on common solutions problems, we refer the readers to [24-38].

In this paper, we first introduce Mann viscosity algorithms via subgradient extragradient techniques, and then establish some strong convergence theorems in Hilbert spaces. It is remarkable that our algorithms involve line-search process.

The following lemmas are useful for the convergence analysis of our algorithms in the sequel.

Lemma 1. [39] Let the operator $A$ be pseudomonotone and continuous on $C$. Given a point $w \in C$. Then the relation holds: $\langle A w, w-y\rangle \leq 0 \forall y \in C \Leftrightarrow\langle A y, w-y\rangle \leq 0 \forall y \in C$.

Lemma 2. [40] Suppose that $\left\{s_{k}\right\}$ is a sequence in $[0,+\infty)$ such that $s_{k+1} \leq t_{k} b_{k}+\left(1-t_{k}\right) s_{k} \forall k \geq 1$, where $\left\{t_{k}\right\}$ and $\left\{b_{k}\right\}$ lie in real line $\boldsymbol{R}:=(-\infty, \infty)$, such that:

(a) $\left\{t_{k}\right\} \subset[0,1]$ and $\sum_{k=1}^{\infty} t_{k}=\infty$;

(b) $\lim \sup _{k \rightarrow \infty} b_{k} \leq 0$ or $\sum_{k=1}^{\infty}\left|t_{k} b_{k}\right|<\infty$. Then $s_{k} \rightarrow 0$ as $k \rightarrow \infty$.

From Ceng et al. [2] it is not difficult to find that the following lemmas hold.

Lemma 3. Let $\Gamma$ be an $\eta$-strictly pseudocontractive self-mapping on $C$. Then $I-\Gamma$ is demiclosed at zero.

Lemma 4. For $l=1, \ldots, N$, let $\Gamma_{l}$ be an $\eta_{l}$-strictly pseudocontractive self-mapping on $C$. Then for $l=1, \ldots, N$, the mapping $\Gamma_{l}$ is an $\eta$-strict pseudocontraction with $\eta=\max \left\{\eta_{l}: 1 \leq l \leq N\right\}$, such that

$$
\left\|\Gamma_{l} u-\Gamma_{l} v\right\| \leq \frac{1+\eta}{1-\eta}\|u-v\| \quad \forall u, v \in C .
$$

Lemma 5. Let $\Gamma$ be an $\eta$-strictly pseudocontractive self-mapping on $C$. Given two reals $\gamma, \beta \in[0,+\infty)$. If $(\gamma+\beta) \eta \leq \gamma$, then $\|\gamma(u-v)+\beta(\Gamma u-\Gamma v)\| \leq(\gamma+\beta)\|u-v\| \forall u, v \in C$. 


\section{Main Results}

Our first algorithm is specified below.

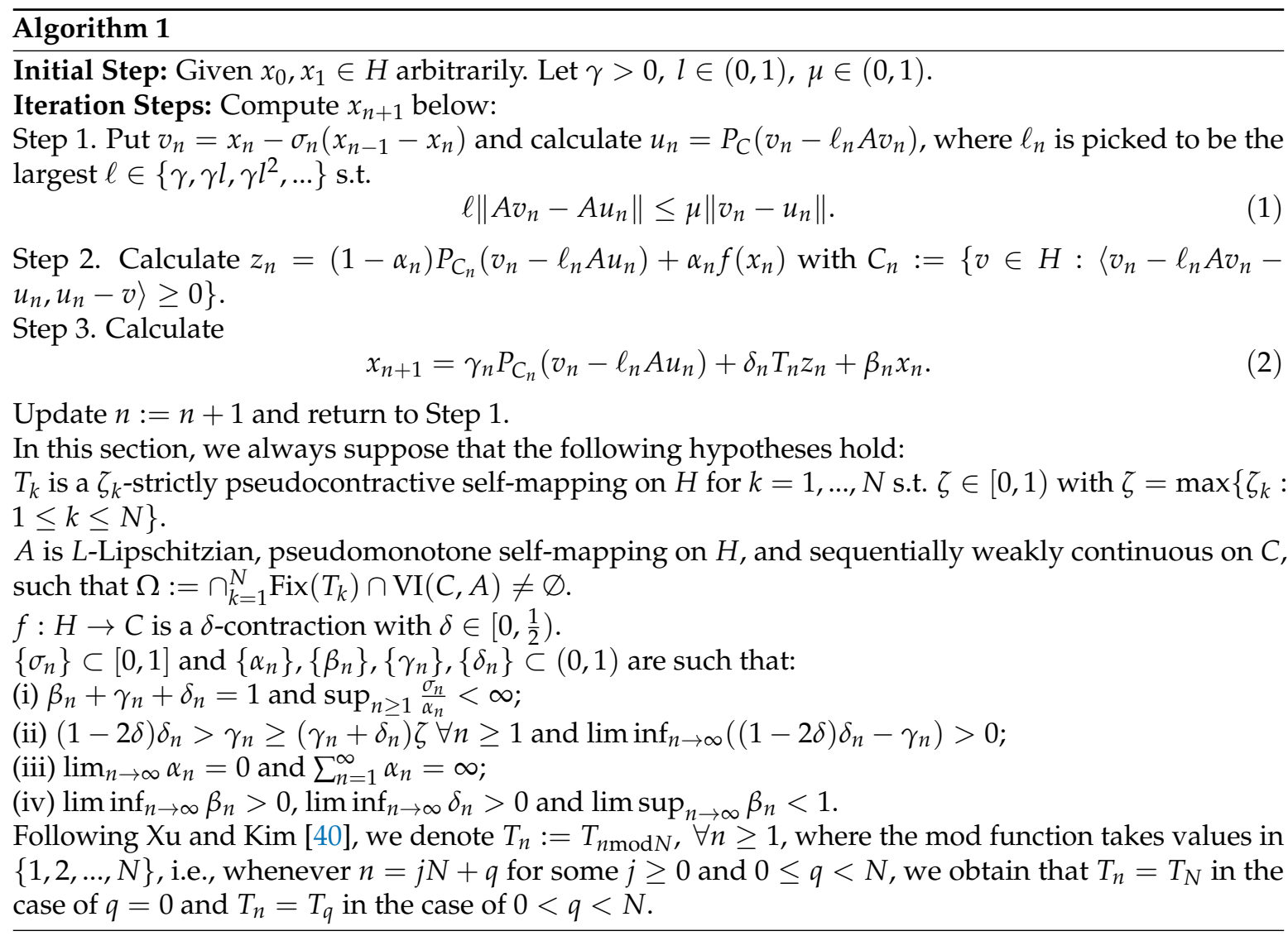

Lemma 6. The Armijo-like search rule (1) is well defined, and $\min \left\{\gamma, \frac{\mu l}{L}\right\} \leq \ell_{n} \leq \gamma$.

Proof. Obviously, (1) holds for all $\gamma l^{m} \leq \frac{\mu}{L}$. So, $\ell_{n}$ is well defined and $\ell_{n} \leq \gamma$. In the case of $\ell_{n}=\gamma$, the inequality is true. In the case of $\ell_{n}<\gamma$, (1) ensures $\left\|A v_{n}-A P_{C}\left(v_{n}-\frac{\ell_{n}}{l} A v_{n}\right)\right\|>$ $\frac{\mu}{\frac{\ell}{l}}\left\|v_{n}-P_{C}\left(v_{n}-\frac{\ell_{n}}{l} A v_{n}\right)\right\|$. The $L$-Lipschitzian property of $A$ yields $\ell_{n}>\frac{\mu l}{L}$.

Lemma 7. Let $\left\{v_{n}\right\},\left\{u_{n}\right\}$ and $\left\{z_{n}\right\}$ be the sequences constructed by Algorithm 1. Then

$$
\begin{aligned}
\left\|z_{n}-\omega\right\|^{2} \leq & \left(1-\alpha_{n}\right)\left\|v_{n}-\omega\right\|^{2}+\alpha_{n} \delta\left\|x_{n}-\omega\right\|^{2}-\left(1-\alpha_{n}\right)(1-\mu)\left[\left\|v_{n}-u_{n}\right\|^{2}\right. \\
& \left.+\left\|h_{n}-u_{n}\right\|^{2}\right]+2 \alpha_{n}\left\langle f \omega-\omega, z_{n}-\omega\right\rangle \forall \omega \in \Omega,
\end{aligned}
$$

where $h_{n}:=P_{C_{n}}\left(v_{n}-\ell_{n} A u_{n}\right) \forall n \geq 1$.

Proof. First, taking an arbitrary $p \in \Omega \subset C \subset C_{n}$, we observe that

$$
\begin{aligned}
2\left\|h_{n}-p\right\|^{2} & \leq 2\left\langle h_{n}-p, v_{n}-\ell_{n} A u_{n}-p\right\rangle \\
& =\left\|h_{n}-p\right\|^{2}+\left\|v_{n}-p\right\|^{2}-\left\|h_{n}-v_{n}\right\|^{2}-2\left\langle\ell_{n} A u_{n}, h_{n}-p\right\rangle .
\end{aligned}
$$

So, it follows that $\left\|v_{n}-p\right\|^{2}-2\left\langle h_{n}-p, \ell_{n} A u_{n}\right\rangle-\left\|h_{n}-v_{n}\right\|^{2} \geq\left\|h_{n}-p\right\|^{2}$, which together with (1), we deduce that $0 \geq\left\langle p-u_{n}, A u_{n}\right\rangle$ and

$$
\begin{aligned}
\left\|h_{n}-p\right\|^{2} & \leq\left\|v_{n}-p\right\|^{2}-\left\|h_{n}-v_{n}\right\|^{2}+2 \ell_{n}\left(\left\langle A u_{n}, p-u_{n}\right\rangle+\left\langle A u_{n}, u_{n}-h_{n}\right\rangle\right) \\
& \leq\left\|v_{n}-p\right\|^{2}-\left\|u_{n}-h_{n}\right\|^{2}-\left\|v_{n}-u_{n}\right\|^{2}+2\left\langle u_{n}-v_{n}+\ell_{n} A u_{n}, u_{n}-h_{n}\right\rangle .
\end{aligned}
$$


Since $h_{n}=P_{C_{n}}\left(v_{n}-\ell_{n} A u_{n}\right)$ with $C_{n}:=\left\{v \in H:\left\langle u_{n}-v_{n}+\ell_{n} A v_{n}, u_{n}-v\right\rangle \leq 0\right\}$, we have $\left\langle u_{n}-v_{n}+\right.$ $\left.\ell_{n} A v_{n}, u_{n}-h_{n}\right\rangle \leq 0$, which together with (1), implies that

$$
\begin{aligned}
2\left\langle u_{n}-v_{n}+\ell_{n} A u_{n}, u_{n}-h_{n}\right\rangle & =2\left\langle u_{n}-v_{n}+\ell_{n} A v_{n}, u_{n}-h_{n}\right\rangle+2 \ell_{n}\left\langle A v_{n}-A u_{n}, h_{n}-u_{n}\right\rangle \\
& \leq 2 \mu\left\|u_{n}-v_{n}\right\|\left\|u_{n}-h_{n}\right\| \leq \mu\left(\left\|v_{n}-u_{n}\right\|^{2}+\left\|h_{n}-u_{n}\right\|^{2}\right) .
\end{aligned}
$$

Therefore, substituting the last inequality for (4), we infer that

$$
\left\|h_{n}-p\right\|^{2} \leq\left\|v_{n}-p\right\|^{2}-(1-\mu)\left\|v_{n}-u_{n}\right\|^{2}-(1-\mu)\left\|h_{n}-u_{n}\right\|^{2} \quad \forall p \in \Omega .
$$

In addition, we have

$$
z_{n}-p=\left(1-\alpha_{n}\right)\left(h_{n}-p\right)+\alpha_{n}(f-I) p+\alpha_{n}\left(f\left(x_{n}\right)-f(p)\right) .
$$

Using the convexity of the function $h(t)=t^{2} \forall t \in \mathbf{R}$, from (5) we get

$$
\begin{aligned}
\left\|z_{n}-p\right\|^{2} \leq & {\left[\alpha_{n} \delta\left\|x_{n}-p\right\|+\left(1-\alpha_{n}\right)\left\|h_{n}-p\right\|\right]^{2}+2 \alpha_{n}\left\langle(f-I) p, z_{n}-p\right\rangle } \\
\leq & \alpha_{n} \delta\left\|x_{n}-p\right\|^{2}+\left(1-\alpha_{n}\right)\left\|h_{n}-p\right\|^{2}+2 \alpha_{n}\left\langle(f-I) p, z_{n}-p\right\rangle \\
\leq & \alpha_{n} \delta\left\|x_{n}-p\right\|^{2}+\left(1-\alpha_{n}\right)\left\|v_{n}-p\right\|^{2}-\left(1-\alpha_{n}\right)(1-\mu)\left[\left\|v_{n}-u_{n}\right\|^{2}\right. \\
& \left.+\left\|h_{n}-u_{n}\right\|^{2}\right]+2 \alpha_{n}\left\langle(f-I) p, z_{n}-p\right\rangle .
\end{aligned}
$$

Lemma 8. Let $\left\{x_{n}\right\},\left\{u_{n}\right\}$, and $\left\{z_{n}\right\}$ be bounded sequences constructed by Algorithm 1. If $x_{n}-x_{n+1} \rightarrow$ $0, v_{n}-u_{n} \rightarrow 0, v_{n}-z_{n} \rightarrow 0$ and $\exists\left\{v_{n_{i}}\right\} \subset\left\{v_{n}\right\}$ s.t. $v_{n_{i}} \rightarrow z \in H$, then $z \in \Omega$.

Proof. According to Algorithm 1, we get $\sigma_{n}\left(x_{n}-x_{n-1}\right)=v_{n}-x_{n} \forall n \geq 1$, and hence $\left\|x_{n}-x_{n-1}\right\| \geq$ $\left\|v_{n}-x_{n}\right\|$. Using the assumption $x_{n}-x_{n+1} \rightarrow 0$, we have

$$
\lim _{n \rightarrow \infty}\left\|v_{n}-x_{n}\right\|=0
$$

So,

$$
\left\|z_{n}-x_{n}\right\| \leq\left\|v_{n}-z_{n}\right\|+\left\|v_{n}-x_{n}\right\| \rightarrow 0 .
$$

Since $\left\{x_{n}\right\}$ is bounded, from $v_{n}=x_{n}-\sigma_{n}\left(x_{n-1}-x_{n}\right)$ we know that $\left\{v_{n}\right\}$ is a bounded vector sequence. According to (5), we obtain that $h_{n}:=P_{C_{n}}\left(v_{n}-\ell_{n} A u_{n}\right)$ is a bounded vector sequence. Also, by Algorithm 1 we get $\alpha_{n} f\left(x_{n}\right)+h_{n}-x_{n}-\alpha_{n} h_{n}=z_{n}-x_{n}$. So, the boundedness of $\left\{x_{n}\right\},\left\{h_{n}\right\}$ guarantees that as $n \rightarrow \infty$,

$$
\left\|h_{n}-x_{n}\right\|=\left\|z_{n}-x_{n}-\alpha_{n} f\left(x_{n}\right)+\alpha_{n} h_{n}\right\| \leq\left\|z_{n}-x_{n}\right\|+\alpha_{n}\left(\left\|f\left(x_{n}\right)\right\|+\left\|h_{n}\right\|\right) \rightarrow 0 .
$$

It follows that

$$
x_{n+1}-z_{n}=\gamma_{n}\left(h_{n}-x_{n}\right)+\delta_{n}\left(T_{n} z_{n}-z_{n}\right)+\left(1-\delta_{n}\right)\left(x_{n}-z_{n}\right),
$$

which immediately yields

$$
\begin{aligned}
\delta_{n}\left\|T_{n} z_{n}-z_{n}\right\| & =\left\|x_{n+1}-x_{n}+x_{n}-z_{n}-\left(1-\delta_{n}\right)\left(x_{n}-z_{n}\right)-\gamma_{n}\left(h_{n}-x_{n}\right)\right\| \\
& =\left\|x_{n+1}-x_{n}+\delta_{n}\left(x_{n}-z_{n}\right)-\gamma_{n}\left(h_{n}-x_{n}\right)\right\| \\
& \leq\left\|x_{n+1}-x_{n}\right\|+\left\|x_{n}-z_{n}\right\|+\left\|h_{n}-x_{n}\right\| .
\end{aligned}
$$


Since $x_{n}-x_{n+1} \rightarrow 0, z_{n}-x_{n} \rightarrow 0, h_{n}-x_{n} \rightarrow 0$ and $\lim \inf _{n \rightarrow \infty} \delta_{n}>0$, we obtain $\left\|z_{n}-T_{n} z_{n}\right\| \rightarrow 0$ as $n \rightarrow \infty$. This further implies that

$$
\begin{aligned}
\left\|x_{n}-T_{n} x_{n}\right\| & \leq\left\|x_{n}-z_{n}\right\|+\left\|z_{n}-T_{n} z_{n}\right\|+\frac{1+\zeta}{1-\zeta}\left\|z_{n}-x_{n}\right\| \\
& \leq \frac{2}{1-\zeta}\left\|x_{n}-z_{n}\right\|+\left\|z_{n}-T_{n} z_{n}\right\| \rightarrow 0 \quad(n \rightarrow \infty)
\end{aligned}
$$

We have $\left\langle v_{n}-\ell_{n} A v_{n}-u_{n}, v-u_{n}\right\rangle \leq 0 \forall v \in C$, and

$$
\left\langle v_{n}-u_{n}, v-u_{n}\right\rangle+\ell_{n}\left\langle A v_{n}, u_{n}-v_{n}\right\rangle \leq \ell_{n}\left\langle A v_{n}, v-v_{n}\right\rangle \quad \forall v \in C
$$

Note that $\ell_{n} \geq \min \left\{\gamma, \frac{\mu l}{L}\right\}$. So, $\liminf _{i \rightarrow \infty}\left\langle A v_{n_{i}}, v-v_{n_{i}}\right\rangle \geq 0 \forall v \in C$. This yields $\liminf _{i \rightarrow \infty}\left\langle A u_{n_{i}}, v-\right.$ $\left.u_{n_{i}}\right\rangle \geq 0 \forall v \in C$. Since $v_{n}-x_{n} \rightarrow 0$ and $v_{n_{i}} \rightarrow z$, we get $x_{n_{i}} \rightarrow z$. We may assume $k=n_{i} \bmod N$ for all $i$. By the assumption $x_{n}-x_{n+k} \rightarrow 0$, we have $x_{n_{i}+j} \rightarrow z$ for all $j \geq 1$. Hence, $\left\|x_{n_{i}+j}-T_{k+j} x_{n_{i}+j}\right\|=$ $\left\|x_{n_{i}+j}-T_{n_{i}+j} x_{n_{i}+j}\right\| \rightarrow 0$. Then the demiclosedness principle implies that $z \in \operatorname{Fix}\left(T_{k+j}\right)$ for all $j$. This ensures that

$$
z \in \bigcap_{k=1}^{N} \operatorname{Fix}\left(T_{k}\right)
$$

We now take a sequence $\left\{\zeta_{i}\right\} \subset(0,1)$ satisfying $\varsigma_{i} \downarrow 0$ as $i \rightarrow \infty$. For all $i \geq 1$, we denote by $m_{i}$ the smallest natural number satisfying

$$
\left\langle A u_{n_{j}}, v-u_{n_{j}}\right\rangle+\varsigma_{i} \geq 0 \quad \forall j \geq m_{i}
$$

Since $\left\{\zeta_{i}\right\}$ is decreasing, it is clear that $\left\{m_{i}\right\}$ is increasing. Noticing that $\left\{u_{m_{i}}\right\} \subset C$ ensures $A u_{m_{i}} \neq$ $0 \forall i \geq 1$, we set $e_{m_{i}}=\frac{A u_{m_{i}}}{\left\|A u_{m_{i}}\right\|^{2}}$, we get $\left\langle A u_{m_{i}}, e_{m_{i}}\right\rangle=1 \forall i \geq 1$. So, from (10) we get $\left\langle A u_{m_{i}}, v+\varsigma_{i} e_{m_{i}}-\right.$ $\left.u_{m_{i}}\right\rangle \geq 0 \forall i \geq 1$. Also, the pseudomonotonicity of $A$ implies $\left\langle A\left(v+\varsigma_{i} e_{m_{i}}\right), v+\varsigma_{i} e_{m_{i}}-u_{m_{i}}\right\rangle \geq 0 \forall i \geq 1$. This immediately leads to

$$
\left\langle A v-A\left(v+\varsigma_{i} h_{m_{i}}\right), v+\varsigma_{i} e_{m_{i}}-u_{m_{i}}\right\rangle-\varsigma_{i}\left\langle A v, h_{m_{i}}\right\rangle \leq\left\langle A v, v-u_{m_{i}}\right\rangle \quad \forall i \geq 1
$$

We claim $\lim _{i \rightarrow \infty} \zeta_{i} e_{m_{i}}=0$. Indeed, from $v_{n_{i}} \rightarrow z$ and $v_{n}-u_{n} \rightarrow 0$, we obtain $u_{n_{i}} \rightarrow z$. So, $\left\{u_{n}\right\} \subset C$ ensures $z \in C$. Also, the sequentially weak continuity of $A$ guarantees that $A u_{n_{i}} \rightarrow A z$. Thus, we have $A z \neq 0$ (otherwise, $z$ is a solution). Moreover, the sequentially weak lower semicontinuity of $\|\cdot\|$ ensures $0<\|A z\| \leq \liminf _{i \rightarrow \infty}\left\|A u_{n_{i}}\right\|$. Since $\left\{u_{m_{i}}\right\} \subset\left\{u_{n_{i}}\right\}$ and $\varsigma_{i} \downarrow 0$ as $i \rightarrow \infty$, we deduce that $0 \leq \limsup _{i \rightarrow \infty}\left\|\varsigma_{i} e_{m_{i}}\right\|=\limsup _{i \rightarrow \infty} \frac{\varsigma_{i}}{\left\|A u_{m_{i}}\right\|} \leq \frac{\limsup _{i \rightarrow \infty} \varsigma_{i}}{\lim \sin _{i \rightarrow \infty}\left\|A u_{n_{i}}\right\|}=0$. Hence we get $\varsigma_{i} e_{m_{i}} \rightarrow 0$.

Finally we claim $z \in \Omega$. In fact, letting $i \rightarrow \infty$, we conclude that the right hand side of (11) tends to zero by the Lipschitzian property of $A$, the boundedness of $\left\{u_{m_{i}}\right\},\left\{h_{m_{i}}\right\}$ and the $\operatorname{limit}_{i \rightarrow \infty} \lim _{i \rightarrow \infty} e_{m_{i}}=0$. Thus, we get $\langle A v, v-z\rangle=\liminf _{i \rightarrow \infty}\left\langle A v, v-u_{m_{i}}\right\rangle \geq 0 \forall v \in C$. So, $z \in \operatorname{VI}(C, A)$. Therefore, from (9) we have $z \in \cap_{k=1}^{N} \operatorname{Fix}\left(T_{k}\right) \cap \operatorname{VI}(C, A)=\Omega$.

Theorem 1. Assume $A(C)$ is bounded. Let $\left\{x_{n}\right\}$ be constructed by Algorithm 1. Then

$$
x_{n} \rightarrow x^{*} \in \Omega \Leftrightarrow\left\{\begin{array}{l}
x_{n}-x_{n+1} \rightarrow 0, \\
\sup _{n \geq 1}\left\|x_{n}-f x_{n}\right\|<\infty
\end{array}\right.
$$

where $x^{*} \in \Omega$ is the unique solution to the hierarchical variational inequality (HVI): $\left\langle(I-f) x^{*}, x^{*}-\omega\right\rangle \leq$ $0, \forall \omega \in \Omega$. 
Proof. Taking into account condition (iv) on $\left\{\gamma_{n}\right\}$, we may suppose that $\left\{\beta_{n}\right\} \subset[a, b] \subset(0,1)$. Applying Banach's Contraction Principle, we obtain existence and uniqueness of a fixed point $x^{*} \in H$ for the mapping $P_{\Omega} \circ f$, which means that $x^{*}=P_{\Omega} f\left(x^{*}\right)$. Hence, the HVI

$$
\left\langle(I-f) x^{*}, x^{*}-\omega\right\rangle \leq 0, \quad \forall \omega \in \Omega
$$

has a unique solution $x^{*} \in \Omega:=\cap_{k=1}^{N} \operatorname{Fix}\left(T_{k}\right) \cap \operatorname{VI}(C, A)$

It is now obvious that the necessity of the theorem is true. In fact, if $x_{n} \rightarrow x^{*} \in \Omega$, then we get $\sup _{n \geq 1}\left\|x_{n}-f\left(x_{n}\right)\right\| \leq \sup _{n \geq 1}\left(\left\|x_{n}-x^{*}\right\|+\left\|x^{*}-f\left(x^{*}\right)\right\|+\left\|f\left(x^{*}\right)-f\left(x_{n}\right)\right\|\right)<\infty$ and

$$
\left\|x_{n}-x_{n+1}\right\| \leq\left\|x_{n}-x^{*}\right\|+\left\|x_{n+1}-x^{*}\right\| \rightarrow 0 \quad(n \rightarrow \infty) .
$$

For the sufficient condition, let us suppose $x_{n}-x_{n+1} \rightarrow 0$ and $\sup _{n \geq 1}\left\|(I-f) x_{n}\right\|<\infty$. The sufficiency of our conclusion is proved in the following steps.

Step 1. We show the boundedness of $\left\{x_{n}\right\}$. In fact, let $p$ be an arbitrary point in $\Omega$. Then $T_{n} p=p \forall n \geq 1$, and

$$
\left\|v_{n}-p\right\|^{2}-(1-\mu)\left\|h_{n}-u_{n}\right\|^{2}-(1-\mu)\left\|v_{n}-u_{n}\right\|^{2} \geq\left\|h_{n}-p\right\|^{2},
$$

which hence leads to

$$
\left\|v_{n}-p\right\| \geq\left\|h_{n}-p\right\| \quad \forall n \geq 1
$$

By the definition of $v_{n}$, we have

$$
\left\|v_{n}-p\right\| \leq\left\|x_{n}-p\right\|+\sigma_{n}\left\|x_{n}-x_{n-1}\right\| \leq\left\|x_{n}-p\right\|+\alpha_{n} \cdot \frac{\sigma_{n}}{\alpha_{n}}\left\|x_{n}-x_{n-1}\right\| .
$$

Noticing $\sup _{n \geq 1} \frac{\sigma_{n}}{\alpha_{n}}<\infty$ and $\sup _{n \geq 1}\left\|x_{n}-x_{n-1}\right\|<\infty$, we obtain that $\sup _{n \geq 1} \frac{\sigma_{n}}{\alpha_{n}}\left\|x_{n}-x_{n-1}\right\|<\infty$. This ensures that $\exists M_{1}>0$ s.t.

$$
\frac{\sigma_{n}}{\alpha_{n}}\left\|x_{n}-x_{n-1}\right\| \leq M_{1} \quad \forall n \geq 1
$$

Combining (14)-(16), we get

$$
\left\|h_{n}-p\right\| \leq\left\|v_{n}-p\right\| \leq\left\|x_{n}-p\right\|+\alpha_{n} M_{1} \quad \forall n \geq 1
$$

Note that $A(C)$ is bounded, $u_{n}=P_{C}\left(v_{n}-\ell_{n} A v_{n}\right), f(H) \subset C \subset C_{n}$ and $h_{n}=P_{C_{n}}\left(v_{n}-\ell_{n} A u_{n}\right)$. Hence we know that $\left\{A u_{n}\right\}$ is bounded. So, from $\sup _{n \geq 1}\left\|(I-f) x_{n}\right\|<\infty$, it follows that

$$
\begin{aligned}
\left\|h_{n}-f\left(x_{n}\right)\right\| & \leq\left\|v_{n}-\ell_{n} A u_{n}-f\left(x_{n}\right)\right\| \\
& \leq\left\|x_{n}-x_{n-1}\right\|+\left\|x_{n}-f\left(x_{n}\right)\right\|+\gamma\left\|A u_{n}\right\| \leq M_{0},
\end{aligned}
$$

where $\exists M_{0}>0$ s.t. $M_{0} \geq \sup _{n \geq 1}\left(\left\|x_{n}-x_{n-1}\right\|+\left\|x_{n}-f\left(x_{n}\right)\right\|+\gamma\left\|A u_{n}\right\|\right)$ (due to the assumption $x_{n}-x_{n+1} \rightarrow 0$ ). Consequently,

$$
\begin{aligned}
\left\|z_{n}-p\right\| & \leq \alpha_{n} \delta\left\|x_{n}-p\right\|+\left(1-\alpha_{n}\right)\left\|h_{n}-p\right\|+\alpha_{n}\|(f-I) p\| \\
& \leq\left(1-\alpha_{n}(1-\delta)\right)\left\|x_{n}-p\right\|+\alpha_{n}\left(M_{1}+\|(f-I) p\|\right),
\end{aligned}
$$

which together with $\left(\gamma_{n}+\delta_{n}\right) \zeta \leq \gamma_{n}$, yields

$$
\begin{aligned}
\left\|x_{n+1}-p\right\| & \leq \beta_{n}\left\|x_{n}-p\right\|+\left(1-\beta_{n}\right)\left\|\frac{1}{1-\beta_{n}}\left[\gamma_{n}\left(z_{n}-p\right)+\delta_{n}\left(T_{n} z_{n}-p\right)\right]\right\|+\gamma_{n}\left\|h_{n}-z_{n}\right\| \\
& \leq \beta_{n}\left\|x_{n}-p\right\|+\left(1-\beta_{n}\right)\left[\left(1-\alpha_{n}(1-\delta)\right)\left\|x_{n}-p\right\|+\alpha_{n}\left(M_{0}+M_{1}+\|(f-I) p\|\right)\right] \\
& =\left[1-\alpha_{n}\left(1-\beta_{n}\right)(1-\delta)\right]\left\|x_{n}-p\right\|+\alpha_{n}\left(1-\beta_{n}\right)(1-\delta) \frac{M_{0}+M_{1}+\|(f-I) p\|}{1-\delta} .
\end{aligned}
$$


This shows that $\left\|x_{n}-p\right\| \leq \max \left\{\left\|x_{1}-p\right\|, \frac{M_{0}+M_{1}+\|(I-f) p\|}{1-\delta}\right\} \forall n \geq 1$. Thus, $\left\{x_{n}\right\}$ is bounded, and so are the sequences $\left\{h_{n}\right\},\left\{v_{n}\right\},\left\{u_{n}\right\},\left\{z_{n}\right\},\left\{T_{n} z_{n}\right\}$.

Step 2. We show that $\exists M_{4}>0$ s.t.

$$
\left(1-\alpha_{n}\right)\left(1-\beta_{n}\right)(1-\mu)\left[\left\|w_{n}-y_{n}\right\|^{2}+\left\|u_{n}-y_{n}\right\|^{2}\right] \leq\left\|x_{n}-p\right\|^{2}-\left\|x_{n+1}-p\right\|^{2}+\alpha_{n} M_{4} .
$$

In fact, using Lemma 7 and the convexity of $\|\cdot\|^{2}$, we get

$$
\begin{aligned}
\left\|x_{n+1}-p\right\|^{2} \leq & \left\|\beta_{n}\left(x_{n}-p\right)+\gamma_{n}\left(z_{n}-p\right)+\delta_{n}\left(T_{n} z_{n}-p\right)\right\|^{2}+2 \gamma_{n} \alpha_{n}\left\langle h_{n}-f\left(x_{n}\right), x_{n+1}-p\right\rangle \\
\leq & \beta_{n}\left\|x_{n}-p\right\|^{2}+\left(1-\beta_{n}\right)\left\|z_{n}-p\right\|^{2}+2\left(1-\beta_{n}\right) \alpha_{n}\left\|h_{n}-f\left(x_{n}\right)\right\|\left\|x_{n+1}-p\right\| \\
\leq & \beta_{n}\left\|x_{n}-p\right\|^{2}+\left(1-\beta_{n}\right)\left\{\alpha_{n} \delta\left\|x_{n}-p\right\|^{2}+\left(1-\alpha_{n}\right)\left\|v_{n}-p\right\|^{2}\right. \\
& \left.-\left(1-\alpha_{n}\right)(1-\mu)\left[\left\|v_{n}-u_{n}\right\|^{2}+\left\|h_{n}-u_{n}\right\|^{2}\right]+\alpha_{n} M_{2}\right\},
\end{aligned}
$$

where $\exists M_{2}>0$ s.t. $M_{2} \geq \sup _{n \geq 1} 2\left(\|(f-I) p\|\left\|z_{n}-p\right\|+\left\|u_{n}-f\left(x_{n}\right)\right\|\left\|x_{n+1}-p\right\|\right)$. Also,

$$
\begin{aligned}
\left\|v_{n}-p\right\|^{2} & \leq\left\|x_{n}-p\right\|^{2}+\alpha_{n}\left(2 M_{1}\left\|x_{n}-p\right\|+\alpha_{n} M_{1}^{2}\right) \\
& \leq\left\|x_{n}-p\right\|^{2}+\alpha_{n} M_{3}
\end{aligned}
$$

where $\exists M_{3}>0$ s.t. $M_{3} \geq \sup _{n \geq 1}\left(2 M_{1}\left\|x_{n}-p\right\|+\beta_{n} M_{1}^{2}\right)$. Substituting (19) for (18), we have

$$
\begin{aligned}
\left\|x_{n+1}-p\right\|^{2} \leq & \beta_{n}\left\|x_{n}-p\right\|^{2}+\left(1-\beta_{n}\right)\left\{\left(1-\alpha_{n}(1-\delta)\right)\left\|x_{n}-p\right\|^{2}+\left(1-\alpha_{n}\right) \alpha_{n} M_{3}\right. \\
& \left.-\left(1-\alpha_{n}\right)(1-\mu)\left[\left\|v_{n}-u_{n}\right\|^{2}+\left\|h_{n}-u_{n}\right\|^{2}\right]+\alpha_{n} M_{2}\right\} \\
\leq & \left\|x_{n}-p\right\|^{2}-\left(1-\alpha_{n}\right)\left(1-\beta_{n}\right)(1-\mu)\left[\left\|v_{n}-u_{n}\right\|^{2}+\left\|h_{n}-u_{n}\right\|^{2}\right]+\alpha_{n} M_{4}
\end{aligned}
$$

where $M_{4}:=M_{2}+M_{3}$. This immediately implies that

$$
\left(1-\alpha_{n}\right)\left(1-\beta_{n}\right)(1-\mu)\left[\left\|v_{n}-u_{n}\right\|^{2}+\left\|h_{n}-u_{n}\right\|^{2}\right] \leq\left\|x_{n}-p\right\|^{2}-\left\|x_{n+1}-p\right\|^{2}+\alpha_{n} M_{4} .
$$

Step 3. We show that $\exists M>0$ s.t.

$$
\begin{aligned}
& \left\|x_{n+1}-p\right\|^{2} \\
& \leq\left[1-\frac{(1-2 \delta) \delta_{n}-\gamma_{n}}{1-\alpha_{n} \gamma_{n}} \alpha_{n}\right]\left\|x_{n}-p\right\|^{2}+\frac{\left[(1-2 \delta) \delta_{n}-\gamma_{n}\right] \alpha_{n}}{1-\alpha_{n} \gamma_{n}} \cdot\left\{\frac{2 \gamma_{n}}{(1-2 \delta) \delta_{n}-\gamma_{n}}\left\|f\left(x_{n}\right)-p\right\|\left\|z_{n}-x_{n+1}\right\|\right. \\
& \quad+\frac{2 \delta \delta_{n}}{(1-2 \delta) \delta_{n}-\gamma_{n}}\left\|f\left(x_{n}\right)-p\right\|\left\|z_{n}-x_{n}\right\|+\frac{\gamma_{n}}{(1-2 \delta) \delta_{n}-\gamma_{n}}\left\langle f(p)-p, x_{n}-p\right\rangle \\
& \left.\quad+\frac{\gamma_{n}+\delta_{n}}{(1-2 \delta) \delta_{n}-\gamma_{n}} \cdot \frac{\sigma_{n}}{\alpha_{n}}\left\|x_{n}-x_{n-1}\right\| 3 M\right\} .
\end{aligned}
$$

In fact, we get

$$
\begin{aligned}
\left\|v_{n}-p\right\|^{2} & \leq\left\|x_{n}-p\right\|^{2}+\sigma_{n}\left\|x_{n}-x_{n-1}\right\|\left(2\left\|x_{n}-p\right\|+\sigma_{n}\left\|x_{n}-x_{n-1}\right\|\right) \\
& \leq\left\|x_{n}-p\right\|^{2}+\sigma_{n}\left\|x_{n}-x_{n-1}\right\| 3 M,
\end{aligned}
$$

where $\exists M>0$ s.t. $M \geq \sup _{n \geq 1}\left\{\left\|x_{n}-p\right\|, \sigma_{n}\left\|x_{n}-x_{n-1}\right\|\right\}$. By Algorithm 1 and the convexity of $\|\cdot\|^{2}$, we have

$$
\begin{aligned}
\left\|x_{n+1}-p\right\|^{2} \leq & \left\|\beta_{n}\left(x_{n}-p\right)+\gamma_{n}\left(z_{n}-p\right)+\delta_{n}\left(T_{n} z_{n}-p\right)\right\|^{2}+2 \gamma_{n} \alpha_{n}\left\langle h_{n}-f\left(x_{n}\right), x_{n+1}-p\right\rangle \\
\leq & \beta_{n}\left\|x_{n}-p\right\|^{2}+\left(1-\beta_{n}\right)\left\|\frac{1}{1-\beta_{n}}\left[\gamma_{n}\left(z_{n}-p\right)+\delta_{n}\left(T_{n} z_{n}-p\right)\right]\right\|^{2} \\
& +2 \gamma_{n} \alpha_{n}\left\langle h_{n}-p, x_{n+1}-p\right\rangle+2 \gamma_{n} \alpha_{n}\left\langle p-f\left(x_{n}\right), x_{n+1}-p\right\rangle,
\end{aligned}
$$

which leads to

$$
\begin{aligned}
\left\|x_{n+1}-p\right\|^{2} \leq & \beta_{n}\left\|x_{n}-p\right\|^{2}+\left(1-\beta_{n}\right)\left[\left(1-\alpha_{n}\right)\left\|h_{n}-p\right\|^{2}+2 \alpha_{n}\left\langle f\left(x_{n}\right)-p, z_{n}-p\right\rangle\right] \\
& +\gamma_{n} \alpha_{n}\left(\left\|h_{n}-p\right\|^{2}+\left\|x_{n+1}-p\right\|^{2}\right)+2 \gamma_{n} \alpha_{n}\left\langle p-f\left(x_{n}\right), x_{n+1}-p\right\rangle .
\end{aligned}
$$


Using (17) and (22) we obtain that $\left\|h_{n}-p\right\|^{2} \leq\left\|x_{n}-p\right\|^{2}+\sigma_{n}\left\|x_{n}-x_{n-1}\right\| 3 M$. Hence,

$$
\begin{aligned}
\left\|x_{n+1}-p\right\|^{2} \leq & {\left[1-\alpha_{n}\left(1-\beta_{n}\right)\right]\left\|x_{n}-p\right\|^{2}+\left(1-\beta_{n}\right)\left(1-\alpha_{n}\right) \sigma_{n}\left\|x_{n}-x_{n-1}\right\| 3 M } \\
& +2 \alpha_{n} \delta_{n}\left\langle f\left(x_{n}\right)-p, z_{n}-p\right\rangle+\gamma_{n} \alpha_{n}\left(\left\|x_{n}-p\right\|^{2}+\left\|x_{n+1}-p\right\|^{2}\right) \\
& +\left(1-\beta_{n}\right) \alpha_{n} \sigma_{n}\left\|x_{n}-x_{n-1}\right\| 3 M+2 \gamma_{n} \alpha_{n}\left\langle f\left(x_{n}\right)-p, z_{n}-x_{n+1}\right\rangle \\
\leq & {\left[1-\alpha_{n}\left(1-\beta_{n}\right)\right]\left\|x_{n}-p\right\|^{2}+2 \gamma_{n} \alpha_{n}\left\|f\left(x_{n}\right)-p\right\|\left\|z_{n}-x_{n+1}\right\| } \\
& +2 \alpha_{n} \delta_{n}\left\langle f\left(x_{n}\right)-p, x_{n}-p\right\rangle+2 \alpha_{n} \delta_{n}\left\langle f\left(x_{n}\right)-p, z_{n}-x_{n}\right\rangle \\
& +\gamma_{n} \alpha_{n}\left(\left\|x_{n}-p\right\|^{2}+\left\|x_{n+1}-p\right\|^{2}\right)+\left(1-\beta_{n}\right) \sigma_{n}\left\|x_{n}-x_{n-1}\right\| 3 M \\
\leq & {\left[1-\alpha_{n}\left(1-\beta_{n}\right)\right]\left\|x_{n}-p\right\|^{2}+2 \gamma_{n} \alpha_{n}\left\|f\left(x_{n}\right)-p\right\|\left\|z_{n}-x_{n+1}\right\| } \\
& +2 \alpha_{n} \delta_{n} \delta\left\|x_{n}-p\right\|^{2}+2 \alpha_{n} \delta_{n}\left\langle f(p)-p, x_{n}-p\right\rangle+2 \alpha_{n} \delta_{n}\left\|f\left(x_{n}\right)-p\right\|\left\|z_{n}-x_{n}\right\| \\
& +\gamma_{n} \alpha_{n}\left(\left\|x_{n}-p\right\|^{2}+\left\|x_{n+1}-p\right\|^{2}\right)+\left(1-\beta_{n}\right) \sigma_{n}\left\|x_{n}-x_{n-1}\right\| 3 M,
\end{aligned}
$$

which immediately yields

$$
\begin{aligned}
\| & x_{n+1}-p \|^{2} \\
\leq & {\left[1-\frac{(1-2 \delta) \delta_{n}-\gamma_{n}}{1-\alpha_{n} \gamma_{n}} \alpha_{n}\right]\left\|x_{n}-p\right\|^{2}+\frac{\left[(1-2 \delta) \delta_{n}-\gamma_{n}\right] \alpha_{n}}{1-\alpha_{n} \gamma_{n}} \cdot\left\{\frac{2 \gamma_{n}}{(1-2 \delta) \delta_{n}-\gamma_{n}}\left\|f\left(x_{n}\right)-p\right\|\left\|z_{n}-x_{n+1}\right\|\right.} \\
& +\frac{2 \delta_{n}}{(1-2 \delta) \delta_{n}-\gamma_{n}}\left\|f\left(x_{n}\right)-p\right\|\left\|z_{n}-x_{n}\right\|+\frac{2 \delta}{(1-2 \delta) \delta_{n}-\gamma_{n}}\left\langle f(p)-p, x_{n}-p\right\rangle \\
& \left.+\frac{\gamma_{n}+\delta_{n}}{(1-2 \delta) \delta_{n}-\gamma_{n}} \cdot \frac{\sigma_{n}}{\alpha_{n}}\left\|x_{n}-x_{n-1}\right\| 3 M\right\} .
\end{aligned}
$$

Step 4. We show that $x_{n} \rightarrow x^{*} \in \Omega$, where $x^{*}$ is the unique solution of (12). Indeed, putting $p=x^{*}$, we infer from (23) that

$$
\begin{aligned}
& \left\|x_{n+1}-x^{*}\right\|^{2} \\
& \leq \\
& \quad\left[1-\frac{(1-2 \delta) \delta_{n}-\gamma_{n}}{1-\alpha_{n} \gamma_{n}} \alpha_{n}\right]\left\|x_{n}-x^{*}\right\|^{2}+\frac{\left[(1-2 \delta) \delta_{n}-\gamma_{n}\right] \alpha_{n}}{1-\alpha_{n} \gamma_{n}} \cdot\left\{\frac{2 \gamma_{n}}{(1-2 \delta) \delta_{n}-\gamma_{n}}\left\|f\left(x_{n}\right)-x^{*}\right\|\left\|z_{n}-x_{n+1}\right\|\right. \\
& \quad+\frac{2 \delta_{n}}{(1-2 \delta) \delta_{n}-\gamma_{n}}\left\|f\left(x_{n}\right)-x^{*}\right\|\left\|z_{n}-x_{n}\right\|+\frac{\gamma_{n}}{(1-2 \delta) \delta_{n}-\gamma_{n}}\left\langle f\left(x^{*}\right)-x^{*}, x_{n}-x^{*}\right\rangle \\
& \left.\quad+\frac{\gamma_{n}+\delta_{n}}{(1-2 \delta) \delta_{n}-\gamma_{n}} \cdot \frac{\sigma_{n}}{\alpha_{n}}\left\|x_{n}-x_{n-1}\right\| 3 M\right\} .
\end{aligned}
$$

It is sufficient to show that $\limsup _{n \rightarrow \infty}\left\langle(f-I) x^{*}, x_{n}-x^{*}\right\rangle \leq 0$. From (21), $x_{n}-x_{n+1} \rightarrow 0, \alpha_{n} \rightarrow 0$ and $\left\{\beta_{n}\right\} \subset[a, b] \subset(0,1)$, we get

$$
\begin{aligned}
& \limsup _{n \rightarrow \infty}\left(1-\alpha_{n}\right)(1-b)(1-\mu)\left[\left\|v_{n}-u_{n}\right\|^{2}+\left\|h_{n}-u_{n}\right\|^{2}\right] \\
& \leq \limsup _{n \rightarrow \infty}\left[\left(\left\|x_{n}-p\right\|+\left\|x_{n+1}-p\right\|\right)\left\|x_{n}-x_{n+1}\right\|+\alpha_{n} M_{4}\right]=0 .
\end{aligned}
$$

This ensures that

$$
\lim _{n \rightarrow \infty}\left\|v_{n}-u_{n}\right\|=0 \text { and } \quad \lim _{n \rightarrow \infty}\left\|h_{n}-u_{n}\right\|=0
$$

Consequently,

$$
\left\|x_{n}-u_{n}\right\| \leq\left\|x_{n}-v_{n}\right\|+\left\|v_{n}-u_{n}\right\| \rightarrow 0 \quad(n \rightarrow \infty) .
$$

Since $z_{n}=\alpha_{n} f\left(x_{n}\right)+\left(1-\alpha_{n}\right) h_{n}$ with $h_{n}:=P_{C_{n}}\left(v_{n}-\ell_{n} A u_{n}\right)$, we get

$$
\begin{aligned}
\left\|z_{n}-u_{n}\right\| & =\left\|\alpha_{n} f\left(x_{n}\right)-\alpha_{n} h_{n}+h_{n}-u_{n}\right\| \\
& \leq \alpha_{n}\left(\left\|f\left(x_{n}\right)\right\|+\left\|h_{n}\right\|\right)+\left\|h_{n}-u_{n}\right\| \rightarrow 0 \quad(n \rightarrow \infty),
\end{aligned}
$$

and hence

$$
\left\|z_{n}-x_{n}\right\| \leq\left\|z_{n}-u_{n}\right\|+\left\|u_{n}-x_{n}\right\| \rightarrow 0 \quad(n \rightarrow \infty) .
$$

Obviously, combining (25) and (26), guarantees that

$$
\left\|v_{n}-z_{n}\right\| \leq\left\|v_{n}-u_{n}\right\|+\left\|u_{n}-z_{n}\right\| \rightarrow 0 \quad(n \rightarrow \infty) .
$$


From the boundedness of $\left\{x_{n}\right\}$, it follows that $\exists\left\{x_{n_{i}}\right\} \subset\left\{x_{n}\right\}$ s.t.

$$
\limsup _{n \rightarrow \infty}\left\langle(f-I) x^{*}, x_{n}-x^{*}\right\rangle=\lim _{i \rightarrow \infty}\left\langle(f-I) x^{*}, x_{n_{i}}-x^{*}\right\rangle .
$$

Since $\left\{x_{n}\right\}$ is bounded, we may suppose that $x_{n_{i}} \rightarrow \tilde{x}$. Hence from (28) we get

$$
\limsup _{n \rightarrow \infty}\left\langle(f-I) x^{*}, x_{n}-x^{*}\right\rangle=\lim _{i \rightarrow \infty}\left\langle(f-I) x^{*}, x_{n_{i}}-x^{*}\right\rangle=\left\langle(f-I) x^{*}, \tilde{x}-x^{*}\right\rangle .
$$

It is easy to see from $v_{n}-x_{n} \rightarrow 0$ and $x_{n_{i}} \rightarrow \tilde{x}$ that $v_{n_{i}} \rightarrow \tilde{x}$. Since $x_{n}-x_{n+1} \rightarrow 0, v_{n}-u_{n} \rightarrow$ $0, v_{n}-z_{n} \rightarrow 0$ and $v_{n_{i}} \rightarrow \tilde{x}$, by Lemma 8 we infer that $\tilde{x} \in \Omega$. Therefore, from (12) and (29) we conclude that

$$
\limsup _{n \rightarrow \infty}\left\langle(f-I) x^{*}, x_{n}-x^{*}\right\rangle=\left\langle(f-I) x^{*}, \tilde{x}-x^{*}\right\rangle \leq 0 .
$$

Note that $\liminf _{n \rightarrow \infty} \frac{(1-2 \delta) \delta_{n}-\gamma_{n}}{1-\alpha_{n} \gamma_{n}}>0$. It follows that $\sum_{n=0}^{\infty} \frac{(1-2 \delta) \delta_{n}-\gamma_{n}}{1-\alpha_{n} \gamma_{n}} \alpha_{n}=\infty$. It is clear that

$$
\begin{gathered}
\limsup _{n \rightarrow \infty}\left\{\frac{2 \gamma_{n}}{(1-2 \delta) \delta_{n}-\gamma_{n}}\left\|f\left(x_{n}\right)-x^{*}\right\|\left\|z_{n}-x_{n+1}\right\|+\frac{2 \delta_{n}}{(1-2 \delta) \delta_{n}-\gamma_{n}}\left\|f\left(x_{n}\right)-x^{*}\right\|\left\|z_{n}-x_{n}\right\|\right. \\
\left.+\frac{2 \delta_{n}}{(1-2 \delta) \delta_{n}-\gamma_{n}}\left\langle f\left(x^{*}\right)-x^{*}, x_{n}-x^{*}\right\rangle+\frac{\gamma_{n}+\delta_{n}}{(1-2 \delta) \delta_{n}-\gamma_{n}} \cdot \frac{\sigma_{n}}{\alpha_{n}}\left\|x_{n}-x_{n-1}\right\| 3 M\right\} \leq 0 .
\end{gathered}
$$

Therefore, by Lemma 2 we immediately deduce that $x_{n} \rightarrow x^{*}$.

Next, we introduce another Mann viscosity algorithm with line-search process by the subgradient extragradient technique.

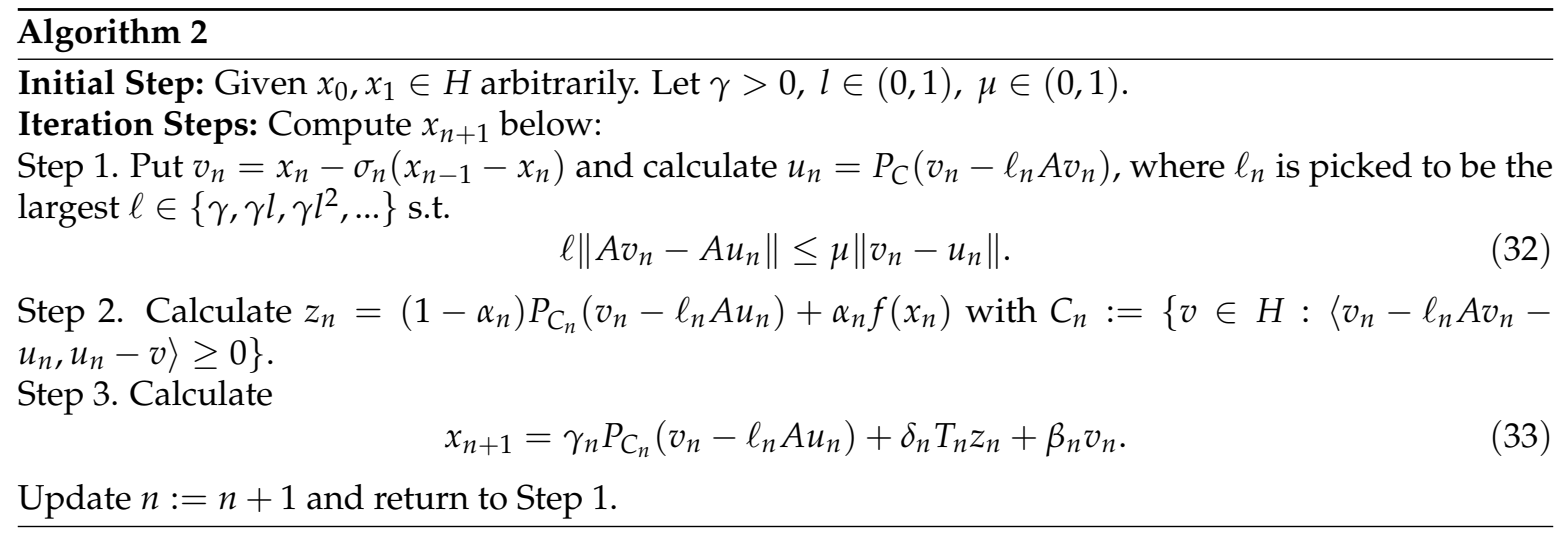

It is remarkable that Lemmas 6, 7 and 8 remain true for Algorithm 2.

Theorem 2. Assume $A(C)$ is bounded. Let $\left\{x_{n}\right\}$ be constructed by Algorithm 2. Then

$$
x_{n} \rightarrow x^{*} \in \Omega \Leftrightarrow\left\{\begin{array}{l}
x_{n}-x_{n+1} \rightarrow 0, \\
\sup _{n \geq 1}\left\|(I-f) x_{n}\right\|<\infty
\end{array}\right.
$$

where $x^{*} \in \Omega$ is the unique solution of the HVI: $\left\langle(I-f) x^{*}, x^{*}-\omega\right\rangle \leq 0, \forall \omega \in \Omega$.

Proof. For the necessity of our proof, we can observe that, by a similar approach to that in the proof of Theorem 1, we obtain that there is a unique solution $x^{*} \in \Omega$ of (12).

We show the sufficiency below. To this aim, we suppose $x_{n}-x_{n+1} \rightarrow 0$ and $\sup _{n \geq 1}\left\|(I-f) x_{n}\right\|<$ $\infty$, and prove the sufficiency by the following steps. 
Step 1. We show the boundedness of $\left\{x_{n}\right\}$. In fact, by the similar inference to that in Step 1 for the proof of Theorem 1, we obtain that (13)-(17) hold. So, using Algorithm 2 and (17) we obtain

$$
\left\|z_{n}-p\right\| \leq\left(1-\alpha_{n}(1-\delta)\right)\left\|x_{n}-p\right\|+\alpha_{n}\left(M_{1}+\|(f-I) p\|\right),
$$

which together with $\left(\gamma_{n}+\delta_{n}\right) \zeta \leq \gamma_{n}$, yields

$$
\begin{aligned}
\left\|x_{n+1}-p\right\| \leq & \beta_{n}\left\|v_{n}-p\right\|+\left(1-\beta_{n}\right)\left\|\frac{1}{1-\beta_{n}}\left[\gamma_{n}\left(z_{n}-p\right)+\delta_{n}\left(T_{n} z_{n}-p\right)\right]\right\|+\gamma_{n}\left\|h_{n}-z_{n}\right\| \\
\leq & \beta_{n}\left(\left\|x_{n}-p\right\|+\alpha_{n} M_{1}\right)+\left(1-\beta_{n}\right)\left[\left(1-\alpha_{n}(1-\delta)\right)\left\|x_{n}-p\right\|\right. \\
& \left.+\alpha_{n}\left(M_{0}+M_{1}+\|(f-I) p\|\right)\right] \\
= & {\left[1-\alpha_{n}\left(1-\beta_{n}\right)(1-\delta)\right]\left\|x_{n}-p\right\|+\alpha_{n}\left(1-\beta_{n}\right)(1-\delta) \frac{M_{0}+\frac{1}{1-\beta_{n}} M_{1}+\|(f-I) p\|}{1-\delta} . }
\end{aligned}
$$

Therefore, we get the boundedness of $\left\{x_{n}\right\}$ and hence the one of sequences $\left\{h_{n}\right\},\left\{v_{n}\right\},\left\{u_{n}\right\},\left\{z_{n}\right\},\left\{T_{n} z_{n}\right\}$.

Step 2. We show that $\exists M_{4}>0$ s.t.

$$
\left(1-\alpha_{n}\right)\left(1-\beta_{n}\right)(1-\mu)\left[\left\|w_{n}-y_{n}\right\|^{2}+\left\|u_{n}-y_{n}\right\|^{2}\right] \leq\left\|x_{n}-p\right\|^{2}-\left\|x_{n+1}-p\right\|^{2}+\alpha_{n} M_{4} .
$$

In fact, by Lemma 7 and the convexity of $\|\cdot\|^{2}$, we get

$$
\begin{aligned}
\left\|x_{n+1}-p\right\|^{2} \leq & \left\|\beta_{n}\left(v_{n}-p\right)+\gamma_{n}\left(z_{n}-p\right)+\delta_{n}\left(T_{n} z_{n}-p\right)\right\|^{2}+2 \gamma_{n} \alpha_{n}\left\langle h_{n}-f\left(x_{n}\right), x_{n+1}-p\right\rangle \\
\leq & \beta_{n}\left\|v_{n}-p\right\|^{2}+\left(1-\beta_{n}\right)\left\|z_{n}-p\right\|^{2}+2\left(1-\beta_{n}\right) \alpha_{n}\left\|h_{n}-f\left(x_{n}\right)\right\|\left\|x_{n+1}-p\right\| \\
\leq & \beta_{n}\left\|v_{n}-p\right\|^{2}+\left(1-\beta_{n}\right)\left\{\alpha_{n} \delta\left\|x_{n}-p\right\|^{2}+\left(1-\alpha_{n}\right)\left\|v_{n}-p\right\|^{2}\right. \\
& \left.-\left(1-\alpha_{n}\right)(1-\mu)\left[\left\|v_{n}-u_{n}\right\|^{2}+\left\|h_{n}-u_{n}\right\|^{2}\right]+\alpha_{n} M_{2}\right\},
\end{aligned}
$$

where $\exists M_{2}>0$ s.t. $M_{2} \geq \sup _{n \geq 1} 2\left(\|(f-I) p\|\left\|z_{n}-p\right\|+\left\|u_{n}-f\left(x_{n}\right)\right\|\left\|x_{n+1}-p\right\|\right)$. Also,

$$
\begin{aligned}
\left\|v_{n}-p\right\|^{2} & \leq\left\|x_{n}-p\right\|^{2}+\alpha_{n}\left(2 M_{1}\left\|x_{n}-p\right\|+\alpha_{n} M_{1}^{2}\right) \\
& \leq\left\|x_{n}-p\right\|^{2}+\alpha_{n} M_{3}
\end{aligned}
$$

where $\exists M_{3}>0$ s.t. $M_{3} \geq \sup _{n \geq 1}\left(2 M_{1}\left\|x_{n}-p\right\|+\beta_{n} M_{1}^{2}\right)$. Substituting (35) for (34), we have

$$
\begin{aligned}
\left\|x_{n+1}-p\right\|^{2} \leq & \beta_{n}\left\|x_{n}-p\right\|^{2}+\left(1-\beta_{n}\right)\left\{\left(1-\alpha_{n}(1-\delta)\right)\left\|x_{n}-p\right\|^{2}+\left(1-\alpha_{n}\right) \alpha_{n} M_{3}\right. \\
& \left.-\left(1-\alpha_{n}\right)(1-\mu)\left[\left\|v_{n}-u_{n}\right\|^{2}+\left\|h_{n}-u_{n}\right\|^{2}\right]+\alpha_{n} M_{2}\right\}+\beta_{n} \alpha_{n} M_{3} \\
= & \left\|x_{n}-p\right\|^{2}-\left(1-\alpha_{n}\right)\left(1-\beta_{n}\right)(1-\mu)\left[\left\|v_{n}-u_{n}\right\|^{2}+\left\|h_{n}-u_{n}\right\|^{2}\right]+\alpha_{n} M_{4}
\end{aligned}
$$

where $M_{4}:=M_{2}+M_{3}$. This ensures that

$$
\left(1-\alpha_{n}\right)\left(1-\beta_{n}\right)(1-\mu)\left[\left\|v_{n}-u_{n}\right\|^{2}+\left\|h_{n}-u_{n}\right\|^{2}\right] \leq\left\|x_{n}-p\right\|^{2}-\left\|x_{n+1}-p\right\|^{2}+\alpha_{n} M_{4} .
$$

Step 3. We show that $\exists M>0$ s.t.

$$
\begin{aligned}
& \left\|x_{n+1}-p\right\|^{2} \\
& \leq \\
& \quad\left[1-\frac{(1-2 \delta) \delta_{n}-\gamma_{n}}{1-\alpha_{n} \gamma_{n}} \alpha_{n}\right]\left\|x_{n}-p\right\|^{2}+\frac{\left[(1-2 \delta) \delta_{n}-\gamma_{n}\right] \alpha_{n}}{1-\alpha_{n} \gamma_{n}} \cdot\left\{\frac{2 \gamma_{n}}{(1-2 \delta) \delta_{n}-\gamma_{n}}\left\|f\left(x_{n}\right)-p\right\|\left\|z_{n}-x_{n+1}\right\|\right. \\
& \quad+\frac{2 \delta_{n}}{(1-2 \delta) \delta_{n}-\gamma_{n}}\left\|f\left(x_{n}\right)-p\right\|\left\|z_{n}-x_{n}\right\|+\frac{\sigma_{n}}{(1-2 \delta) \delta_{n}-\gamma_{n}}\left\langle f(p)-p, x_{n}-p\right\rangle \\
& \left.\quad+\frac{1}{(1-2 \delta) \delta_{n}-\gamma_{n}} \cdot \frac{\sigma_{n}}{\alpha_{n}}\left\|x_{n}-x_{n-1}\right\| 3 M\right\} .
\end{aligned}
$$

In fact, we get

$$
\begin{aligned}
\left\|v_{n}-p\right\|^{2} & \leq\left\|x_{n}-p\right\|^{2}+\sigma_{n}\left\|x_{n}-x_{n-1}\right\|\left(2\left\|x_{n}-p\right\|+\sigma_{n}\left\|x_{n}-x_{n-1}\right\|\right) \\
& \leq\left\|x_{n}-p\right\|^{2}+\sigma_{n}\left\|x_{n}-x_{n-1}\right\| 3 M
\end{aligned}
$$


where $\exists M>0$ s.t. $M \geq \sup _{n \geq 1}\left\{\left\|x_{n}-p\right\|, \sigma_{n}\left\|x_{n}-x_{n-1}\right\|\right\}$. Using Algorithm 1 and the convexity of $\|\cdot\|^{2}$, we get

$$
\begin{aligned}
\left\|x_{n+1}-p\right\|^{2} \leq & \left\|\beta_{n}\left(v_{n}-p\right)+\gamma_{n}\left(z_{n}-p\right)+\delta_{n}\left(T_{n} z_{n}-p\right)\right\|^{2}+2 \gamma_{n} \alpha_{n}\left\langle h_{n}-f\left(x_{n}\right), x_{n+1}-p\right\rangle \\
\leq & \beta_{n}\left\|v_{n}-p\right\|^{2}+\left(1-\beta_{n}\right)\left\|\frac{1}{1-\beta_{n}}\left[\gamma_{n}\left(z_{n}-p\right)+\delta_{n}\left(T_{n} z_{n}-p\right)\right]\right\|^{2} \\
& +2 \gamma_{n} \alpha_{n}\left\langle h_{n}-p, x_{n+1}-p\right\rangle+2 \gamma_{n} \alpha_{n}\left\langle p-f\left(x_{n}\right), x_{n+1}-p\right\rangle,
\end{aligned}
$$

which leads to

$$
\begin{array}{cc}
\left\|x_{n+1}-p\right\|^{2} \quad \beta_{n}\left\|v_{n}-p\right\|^{2}+\left(1-\beta_{n}\right)\left[\left(1-\alpha_{n}\right)\left\|h_{n}-p\right\|^{2}+2 \alpha_{n}\left\langle f\left(x_{n}\right)-p, z_{n}-p\right\rangle\right] \\
+\gamma_{n} \alpha_{n}\left(\left\|h_{n}-p\right\|^{2}+\left\|x_{n+1}-p\right\|^{2}\right)+2 \gamma_{n} \alpha_{n}\left\langle p-f\left(x_{n}\right), x_{n+1}-p\right\rangle .
\end{array}
$$

Using (17) and (38) we deduce that $\left\|h_{n}-p\right\|^{2} \leq\left\|v_{n}-p\right\|^{2} \leq\left\|x_{n}-p\right\|^{2}+\sigma_{n}\left\|x_{n}-x_{n-1}\right\| 3 M$. Hence,

$$
\begin{aligned}
\left\|x_{n+1}-p\right\|^{2} \leq & {\left[1-\alpha_{n}\left(1-\beta_{n}\right)\right]\left\|x_{n}-p\right\|^{2}+\left[1-\alpha_{n}\left(1-\beta_{n}\right)\right] \sigma_{n}\left\|x_{n}-x_{n-1}\right\| 3 M } \\
& +2 \alpha_{n} \delta_{n}\left\langle f\left(x_{n}\right)-p, z_{n}-p\right\rangle+\gamma_{n} \alpha_{n}\left(\left\|x_{n}-p\right\|^{2}+\left\|x_{n+1}-p\right\|^{2}\right) \\
& +\left(1-\beta_{n}\right) \alpha_{n} \sigma_{n}\left\|x_{n}-x_{n-1}\right\| 3 M+2 \gamma_{n} \alpha_{n}\left\langle f\left(x_{n}\right)-p, z_{n}-x_{n+1}\right\rangle \\
\leq & {\left[1-\alpha_{n}\left(1-\beta_{n}\right)\right]\left\|x_{n}-p\right\|^{2}+2 \gamma_{n} \alpha_{n}\left\|f\left(x_{n}\right)-p\right\|\left\|z_{n}-x_{n+1}\right\| } \\
& +2 \alpha_{n} \delta_{n}\left\langle f\left(x_{n}\right)-p, x_{n}-p\right\rangle+2 \alpha_{n} \delta_{n}\left\langle f\left(x_{n}\right)-p, z_{n}-x_{n}\right\rangle \\
& +\gamma_{n} \alpha_{n}\left(\left\|x_{n}-p\right\|^{2}+\left\|x_{n+1}-p\right\|^{2}\right)+\sigma_{n}\left\|x_{n}-x_{n-1}\right\| 3 M \\
\leq & {\left[1-\alpha_{n}\left(1-\beta_{n}\right)\right]\left\|x_{n}-p\right\|^{2}+2 \gamma_{n} \alpha_{n}\left\|f\left(x_{n}\right)-p\right\|\left\|z_{n}-x_{n+1}\right\| } \\
& +2 \alpha_{n} \delta_{n} \delta\left\|x_{n}-p\right\|^{2}+2 \alpha_{n} \delta_{n}\left\langle f(p)-p, x_{n}-p\right\rangle+2 \alpha_{n} \delta_{n}\left\|f\left(x_{n}\right)-p\right\|\left\|z_{n}-x_{n}\right\| \\
& +\gamma_{n} \alpha_{n}\left(\left\|x_{n}-p\right\|^{2}+\left\|x_{n+1}-p\right\|^{2}\right)+\sigma_{n}\left\|x_{n}-x_{n-1}\right\| 3 M,
\end{aligned}
$$

which immediately yields

$$
\begin{aligned}
\| & x_{n+1}-p \|^{2} \\
\leq & {\left[1-\frac{(1-2 \delta) \delta_{n}-\gamma_{n}}{1-\alpha_{n} \gamma_{n}} \alpha_{n}\right]\left\|x_{n}-p\right\|^{2}+\frac{\left[(1-2 \delta) \delta_{n}-\gamma_{n}\right] \alpha_{n}}{1-\alpha_{n} \gamma_{n}} \cdot\left\{\frac{2 \gamma_{n}}{(1-2 \delta) \delta_{n}-\gamma_{n}}\left\|f\left(x_{n}\right)-p\right\|\left\|z_{n}-x_{n+1}\right\|\right.} \\
& +\frac{2 \delta_{n}}{(1-2 \delta) \delta_{n}-\gamma_{n}}\left\|f\left(x_{n}\right)-p\right\|\left\|z_{n}-x_{n}\right\|+\frac{\sigma_{n}}{(1-2 \delta) \delta_{n}-\gamma_{n}}\left\langle f(p)-p, x_{n}-p\right\rangle \\
& \left.+\frac{1}{(1-2 \delta) \delta_{n}-\gamma_{n}} \cdot \frac{\sigma_{n}}{\alpha_{n}}\left\|x_{n}-x_{n-1}\right\| 3 M\right\} .
\end{aligned}
$$

Step 4. In order to show that $x_{n} \rightarrow x^{*} \in \Omega$, which is the unique solution of (12), we can follow a similar method to that in Step 4 for the proof of Theorem 1.

Finally, we apply our main results to solve the VIP and common fixed point problem (CFPP) in the following illustrating example.

The starting point $x_{0}=x_{1}$ is randomly picked in the real line. Put $f(u)=\frac{1}{8} \sin u, \gamma=l=\mu=$ $\frac{1}{2}, \sigma_{n}=\alpha_{n}=\frac{1}{n+1}, \beta_{n}=\frac{1}{3}, \gamma_{n}=\frac{1}{6}$ and $\delta_{n}=\frac{1}{2}$.

We first provide an example of Lipschitzian, pseudomonotone self-mapping $A$ satisfying the boundedness of $A(C)$ and strictly pseudocontractive self-mapping $T_{1}$ with $\Omega=\operatorname{Fix}\left(T_{1}\right) \cap \operatorname{VI}(C, A) \neq$ $\varnothing$. Let $C=[-1,2]$ and $H$ be the real line with the inner product $\langle a, b\rangle=a b$ and induced norm $\|\cdot\|=|\cdot|$. Then $f$ is a $\delta$-contractive map with $\delta=\frac{1}{8} \in\left[0, \frac{1}{2}\right)$ and $f(H) \subset C$ because $\|f(u)-f(v)\|=$ $\frac{1}{8}\|\sin u-\sin v\| \leq \frac{1}{8}\|u-v\|$ for all $u, v \in H$.

Let $A: H \rightarrow H$ and $T_{1}: H \rightarrow H$ be defined as $A u:=\frac{1}{1+|\sin u|}-\frac{1}{1+|u|}$, and $T_{1} u:=\frac{1}{2} u-\frac{3}{8} \sin u$ for all $u \in H$. Now, we first show that $A$ is $L$-Lipschitzian, pseudomonotone operator with $L=2$, such that $A(C)$ is bounded. In fact, for all $u, v \in H$ we get

$$
\begin{aligned}
\|A u-A v\| & \leq\left|\frac{1}{1+\|u\|}-\frac{1}{1+\|v\|}\right|+\left|\frac{1}{1+\|\sin u\|}-\frac{1}{1+\|\sin v\|}\right| \\
& =\left|\frac{\|v\|-\|u\|}{(1+\|u\|)(1+\|v\|)}\right|+\left|\frac{\|\sin v\|-\|\sin u\|}{(1+\|\sin u\|)(1+\|\sin v\|)}\right| \\
& \leq \frac{\|u-v\|}{(1+\|u\|)(1+\|v\|)}+\frac{\|\sin u-\sin v\|}{(1+\|\sin u\|)(1+\|\sin v\|)} \\
& \leq 2\|u-v\| .
\end{aligned}
$$


This implies that $A$ is 2-Lipschitzian. Next, we show that $A$ is pseudomonotone. For any given $u, v \in H$, it is clear that the relation holds:

$$
\langle A u, u-v\rangle=\left(\frac{1}{1+|\sin u|}-\frac{1}{1+|u|}\right)(u-v) \leq 0 \Rightarrow\langle A v, u-v\rangle=\left(\frac{1}{1+|\sin v|}-\frac{1}{1+|v|}\right)(u-v) \leq 0
$$

Furthermore, it is easy to see that $T_{1}$ is strictly pseudocontractive with constant $\zeta_{1}=\frac{1}{4}$. In fact, we observe that for all $u, v \in H$,

$$
\left\|T_{1} u-T_{1} v\right\| \leq \frac{1}{2}\|u-v\|+\frac{3}{8}\|\sin u-\sin v\| \leq\|u-v\|+\frac{1}{4}\left\|\left(I-T_{1}\right) u-\left(I-T_{1}\right) v\right\| .
$$

It is clear that $\left(\gamma_{n}+\delta_{n}\right) \zeta_{1}=\left(\frac{1}{6}+\frac{1}{2}\right) \cdot \frac{1}{4} \leq \frac{1}{6}=\gamma_{n}<(1-2 \delta) \delta_{n}=\left(1-2 \cdot \frac{1}{8}\right) \frac{1}{2}=\frac{3}{8}$ for all $n \geq 1$. In addition, it is clear that $\operatorname{Fix}\left(T_{1}\right)=\{0\}$ and $A 0=0$ because the derivative $d\left(T_{1} u\right) / d u=\frac{1}{2}-\frac{3}{8} \cos u>0$. Therefore, $\Omega=\{0\} \neq \varnothing$. In this case, Algorithm 1 can be rewritten below:

$$
\left\{\begin{array}{l}
v_{n}=x_{n}-\frac{1}{n+1}\left(x_{n-1}-x_{n}\right) \\
u_{n}=P_{C}\left(v_{n}-\ell_{n} A v_{n}\right) \\
z_{n}=\frac{1}{n+1} f\left(x_{n}\right)+\frac{n}{n+1} P_{C_{n}}\left(v_{n}-\ell_{n} A u_{n}\right), \\
x_{n+1}=\frac{1}{3} x_{n}+\frac{1}{6} P_{C_{n}}\left(v_{n}-\ell_{n} A u_{n}\right)+\frac{1}{2} T_{1} z_{n} \quad \forall n \geq 1
\end{array}\right.
$$

with $\left\{C_{n}\right\}$ and $\left\{\ell_{n}\right\}$, selected as in Algorithm 1. Then, by Theorem 1 , we know that $x_{n} \rightarrow 0 \in \Omega$ iff $x_{n}-x_{n+1} \rightarrow 0(n \rightarrow \infty)$ and $\sup _{n \geq 1}\left|x_{n}-\frac{1}{8} \sin x_{n}\right|<\infty$.

On the other hand, Algorithm 2 can be rewritten below:

$$
\left\{\begin{array}{l}
v_{n}=x_{n}-\frac{1}{n+1}\left(x_{n-1}-x_{n}\right), \\
u_{n}=P_{C}\left(v_{n}-\ell_{n} A v_{n}\right), \\
z_{n}=\frac{1}{n+1} f\left(x_{n}\right)+\frac{n}{n+1} P_{C_{n}}\left(v_{n}-\ell_{n} A u_{n}\right), \\
x_{n+1}=\frac{1}{3} v_{n}+\frac{1}{6} P_{C_{n}}\left(v_{n}-\ell_{n} A u_{n}\right)+\frac{1}{2} T_{1} z_{n} \quad \forall n \geq 1,
\end{array}\right.
$$

with $\left\{C_{n}\right\}$ and $\left\{\ell_{n}\right\}$, selected as in Algorithm 2. Then, by Theorem 2 , we know that $x_{n} \rightarrow 0 \in \Omega$ iff $x_{n}-x_{n+1} \rightarrow 0(n \rightarrow \infty)$ and $\sup _{n \geq 1}\left|x_{n}-\frac{1}{8} \sin x_{n}\right|<\infty$.

Author Contributions: All authors contributed equally to this manuscript.

Funding: This research was partially supported by the Innovation Program of Shanghai Municipal Education Commission (15ZZ068), Ph.D. Program Foundation of Ministry of Education of China (20123127110002) and Program for Outstanding Academic Leaders in Shanghai City (15XD1503100).

Conflicts of Interest: The authors certify that they have no affiliations with or involvement in any organization or entity with any financial or non-financial interest in the subject matter discussed in this manuscript.

\section{References}

1. Browder, F.E.; Petryshyn, W.V. Construction of fixed points of nonlinear mappings in Hilbert space. J. Math. Anal. Appl. 1967, 1967, 197-228. [CrossRef]

2. Ceng, L.C.; Kong, Z.R.; Wen, C.F. On general systems of variational inequalities. Comput. Math. Appl. 2013, 66, 1514-1532. [CrossRef]

3. Nguyen, L.V. Some results on strongly pseudomonotone quasi-variational inequalities. Set-Valued Var. Anal. 2019. [CrossRef]

4. Bin Dehaish, B.A. Weak and strong convergence of algorithms for the sum of two accretive operators with applications. J. Nonlinear Convex Anal. 2015, 16, 1321-1336.

5. Qin, X.; Cho, S.Y.; Wang, L. Strong convergence of an iterative algorithm involving nonlinear mappings of nonexpansive and accretive type. Optimization 2018, 67, 1377-1388. [CrossRef]

6. Liu, L. A hybrid steepest method for solving split feasibility problems inovling nonexpansive mappings. J. Nonlinear Convex Anal. 2019, 20, 471-488. 
7. Ceng, L.C.; Ansari, Q.H.; Yao, J.C. Some iterative methods for finding fixed points and for solving constrained convex minimization problems. Nonlinear Anal. 2011 74, 5286-5302. [CrossRef]

8. Ceng, L.C.; Ansari, Q.H.; Yao, J.C. An extragradient method for solving split feasibility and fixed point problems. Comput. Math. Appl. 2012, 64, 633-642. [CrossRef]

9. Ceng, L.C.; Ansari, Q.H.; Yao, J.C. Relaxed extragradient methods for finding minimum-norm solutions of the split feasibility problem. Nonlinear Anal. 2012, 75, 2116-2125. [CrossRef]

10. Qin, X.; Cho, S.Y.; Yao, J.C. Weak and strong convergence of splitting algorithms in Banach spaces. Optimization 2019. [CrossRef]

11. Cho, S.Y.; Li, W.; Kang, S.M. Convergence analysis of an iterative algorithm for monotone operators. J. Inequal. Appl. 2013, 2013, 199. [CrossRef]

12. Cho, S.Y. Strong convergence analysis of a hybrid algorithm for nonlinear operators in a Banach space. J. Appl. Anal. Comput. 2018, 8, 19-31.

13. Ceng, L.C.; Petrusel, A.; Yao, J.C.; Yao, Y. Hybrid viscosity extragradient method for systems of variational inequalities, fixed points of nonexpansive mappings, zero points of accretive operators in Banach spaces. Fixed Point Theory 2018, 19, 487-501. [CrossRef]

14. Ceng, L.C.; Petrusel, A.; Yao, J.C.; Yao, Y. Systems of variational inequalities with hierarchical variational inequality constraints for Lipschitzian pseudocontractions. Fixed Point Theory 2019, 20, 113-134. [CrossRef]

15. Cho, S.Y.; Kang, S.M. Approximation of fixed points of pseudocontraction semigroups based on a viscosity iterative process. Appl. Math. Lett. 2011, 24, 224-228. [CrossRef]

16. Cho, S.Y.; Kang, S.M. Approximation of common solutions of variational inequalities via strict pseudocontractions. Acta Math. Sci. 2012, 32, 1607-1618. [CrossRef]

17. Ceng, L.C.; Yuan, Q. Hybrid Mann viscosity implicit iteration methods for triple hierarchical variational inequalities, systems of variational inequalities and fixed point problems. Mathematics 2019, 7, 142. [CrossRef]

18. Qin, X.; Yao, J.C. Projection splitting algorithms for nonself operators. J. Nonlinear Convex Anal. 2017, 18, 925-935.

19. Qin, X.; Yao, J.C. Weak convergence of a Mann-like algorithm for nonexpansive and accretive operators. J. Inequal. Appl. 2016, 2016, 232. [CrossRef]

20. Ceng, L.C.; Wong, M.M.; Yao, J.C. A hybrid extragradient-like approximation method with regularization for solving split feasibility and fixed point problems. J. Nonlinear Convex Anal. 2013, 14, 163-182.

21. Kraikaew, R.; Saejung, S. Strong convergence of the Halpern subgradient extragradient method for solving variational inequalities in Hilbert spaces. J. Optim. Theory Appl. 2014, 163, 399-412. [CrossRef]

22. Thong, D.V.; Hieu, D.V. Modified subgradient extragradient method for variational inequality problems. Numer. Algorithms 2018, 79, 597-610. [CrossRef]

23. Thong, D.V.; Hieu, D.V. Inertial subgradient extragradient algorithms with line-search process for solving variational inequality problems and fixed point problems. Numer. Algorithms 2019, 80, 1283-1307. [CrossRef]

24. Takahahsi, W.; Yao, J.C. The split common fixed point problem for two finite families of nonlinear mappings in Hilbert spaces. J. Nonlinear Convex Anal. 2019, 20, 173-195.

25. Ansari, Q.H.; Babu, F.; Yao, J.C. Regularization of proximal point algorithms in Hadamard manifolds. J. Fixed Point Theory Appl. 2019, 21, 25. [CrossRef]

26. Qin, X.; Cho, S.Y.; Wang, L. Iterative algorithms with errors for zero points of m-accretive operators. Fixed Point Theory Appl. 2013, 2013, 148. [CrossRef]

27. Takahashi, W.; Wen, C.F.; Yao, J.C. The shrinking projection method for a finite family of demimetric mappings with variational inequality problems in a Hilbert space. Fixed Point Theory 2018, 19, 407-419. [CrossRef]

28. Zhao, X.; Ng, K.F.; Li, C.; Yao, J.C. Linear regularity and linear convergence of projection-based methods for solving convex feasibility problems. Appl. Math. Optim. 2018, 78, 613-641. [CrossRef]

29. Cho, S.Y.; Bin Dehaish, B.A. Weak convergence of a splitting algorithm in Hilbert spaces. J. Appl. Anal. Comput. 2017, 7, 427-438.

30. Cho, S.Y.; Qin, X. On the strong convergence of an iterative process for asymptotically strict pseudocontractions and equilibrium problems. Appl. Math. Comput. 2014, 235, 430-438. [CrossRef]

31. Chang, S.S.; Wen, C.F.; Yao, J.C. Common zero point for a finite family of inclusion problems of accretive mappings in Banach spaces. Optimization 2018, 67, 1183-1196. [CrossRef] 
32. Chang, S.S.; Wen, C.F.; Yao, J.C. Zero point problem of accretive operators in Banach spaces. Bull. Malays. Math. Sci. Soc. 2019, 42, 105-118. [CrossRef]

33. Qin, X.; Cho, S.Y.; Wang, L. A regularization method for treating zero points of the sum of two monotone operators. Fixed Point Theory Appl. 2014, 2014, 75. [CrossRef]

34. Ceng, L.C.; Ansari, Q.H.; Wong, N.C.; Yao, J.C. An extragradient-like approximation method for variational inequalities and fixed point problems. Fixed Point Theory Appl. 2011, 2011, 18. [CrossRef]

35. Ceng, L.C.; Petrusel, A.; Yao, J.C. Composite viscosity approximation methods for equilibrium problem, variational inequality and common fixed points. J. Nonlinear Convex Anal. 2014, 15, 219-240.

36. Ceng, L.C.; Plubtieng, S.; Wong, M.M.; Yao, J.C. System of variational inequalities with constraints of mixed equilibria, variational inequalities, and convex minimization and fixed point problems. J. Nonlinear Convex Anal. 2015, 16, 385-421.

37. Ceng, L.C.; Gupta, H.; Ansari, Q.H. Implicit and explicit algorithms for a system of nonlinear variational inequalities in Banach spaces. J. Nonlinear Convex Anal. 2015, 16, 965-984.

38. Ceng, L.C.; Guu, S.M.; Yao, J.C. Hybrid iterative method for finding common solutions of generalized mixed equilibrium and fixed point problems. Fixed Point Theory Appl. 2012, 2012, 92. [CrossRef]

39. Cottle, R.W.; Yao, J.C. Pseudo-monotone complementarity problems in Hilbert space. J. Optim. Theory Appl. 1992, 75, 281-295. [CrossRef]

40. Xu, H.K.; Kim, T.H. Convergence of hybrid steepest-descent methods for variational inequalities. J. Optim. Theory Appl. 2003, 119, 185-201. [CrossRef]

(C) 2019 by the authors. Licensee MDPI, Basel, Switzerland. This article is an open access article distributed under the terms and conditions of the Creative Commons Attribution (CC BY) license (http:/ / creativecommons.org/licenses/by/4.0/). 